\title{
Selection of Digital Filter for Microprocessor Protection Relays
}

\author{
Denis B. Solovev \\ Vladivostok Branch of Russian Customs Academy, Far Eastern Federal University, Russia
}

\begin{tabular}{l} 
Article Info \\
\hline Article history: \\
Received Jan 15, 2018 \\
Revised Mar 13, 2018 \\
Accepted Mar 28, 2018 \\
\hline
\end{tabular}

Keyword:

Current measurement

Digital filters

Digital measurements

Electric variables control

Modeling

Protective relaying

\begin{abstract}
The article considers some issues related to replacement of electromechanical relays used for protection of power facilities with microprocessor relays. One of the urgent problems connected with implementation of microprocessor overcurrent protections is how to use current transducers other than usual current transformers and in particular Rogowski coils that become more and more widespread. In the article are compared twelve methods of synthesis of a digital filter basing on the analog prototype - second-order integrating filter. The bilinear filter and Boxer-Thaler filters are analyzed in respect to their use in microprocessor relays. Basing on the research results a technique for selection of parameters of digital integrating filters for microprocessor relays is proposed. Simulation results show that Boxer-Thaler and bilinear filters have better accuracy during transient current measurements than the analog filter. The study allows concluding that in many cases the digital second-order bilinear filter is the best choice for use in microprocessor relays.
\end{abstract}

Copyright (C) 2018 Institute of Advanced Engineering and Science. All rights reserved.

\section{Corresponding Author:}

Denis B. Solovev,

Training center, Vladivostok Branch of Russian Customs Academy,

Engineering school, Far Eastern Federal University,

8, Sukhanov St., Vladivostok, 690950, Russia.

Email: solovev.db@dvfu.ru

\section{INTRODUCTION}

Presently microprocessor-based protective relaying is becoming more and more widespread and continues to force out conventional relaying equipment. It is confirmed by the fact that for the last 10 years microprocessor-based relaying has been the mandatory subject for all students in the pertaining fields.

The transition to digital relaying is inevitable [1], [2]. It is known that such large international relay vendors as ABB, General Electric, Siemens, Alstom may soon stop the production of electromechanical relays. This fact is mainly explained by the argument that it is much more profitable to manufacture complex microprocessor-based relays than their electromechanical or solid-state counterparts [1]-[3].

It should be noted that transition to microprocessor-based systems is explained by modern requirements of main purchasers of protective systems (large industrial enterprises). For example, energy and resource-saving efficiency improving programs are now introduced in the Russian Federation, in accordance with the strategy of development of large industrial enterprises. Increasing energy-saving suggests integration of automated utility metering systems at enterprises. In connection with high costs of organizing an automated metering system in distribution networks of large industrial enterprises, the customers' main requirement to such a system is execution of not only its inherent functions, but also power equipment monitoring and protection system functions.

An automated metering and monitoring (protection) system is a distributed information system that monitors and controls operation and power consumption of devices involved in enterprise processes. An important function of such systems is possibility of using MP-based protections against probable emergency conditions. 
Digital protection and diagnostic systems enable significant reduction of power equipment postemergency downtime, which is achieved through immediate notification of the chief power engineer and electrical repair services of the enterprise about the causes of the existing emergency situation.

Most specialists point out that transition to digital methods of information processing in relay protection devices did not lead to development of any new electrical installation protection design principles, but significantly improved relay performance. The latter makes digital devices competitive on the relay protection market [4]-[6].

The main advantage of MP-based protection systems is the possibility of recording and then reproducing for analysis of emergency situations, conditions immediately preceding the emergency and during it. Digital relay protection devices enable transmitting all information about their state to remote dispatch operator stations over special communication channels as well as changing configuration of protection sets and operation settings using a connected computer. Modern MP-based protection devices are more sensitive that their electromechanical counterparts. Besides, one small-sized digital relay can replace a whole group of common electromechanical relays. This is especially true for complex protections, for example, remote ones. All this, together with high reliability, makes it possible to significantly reduce damage caused by power interruptions.

\section{SPECIAL FEATURES OF SIGNAL CONVERSION IN MP-BASED PROTECTIONS}

Most MP-based protections have only analog inputs of voltage and current circuits [7], [8]. Signal from the protected circuit is received from the symmetrical component filter or directly from primary current converters, which are mostly current transformers (CT) (owing to which MP-based systems have some drawbacks). Analog signals are converted to digital ones by means of an analog/digital converter (ADC), which is the most vulnerable point of the whole MP-based protection system as it can distort or block information transmitted by CT or other primary current converters.

At present, there are hundreds of models of MP-based protection devices produced by dozens of different manufacturers on the power electronics market. Almost every type of MP-based protection units has its own enclosure that significantly differs from enclosures of another type. What remains the same is only the number and type of basic standard units enabling high-performance operation of MP-based protection and instrument systems.

Based on the analog-to-digital signal conversion principle, one can distinguish three structural diagrams of MP-based relay protection IEDs for distribution networks of industrial enterprises (Figure 1) [3]-[5], [9]-[11].

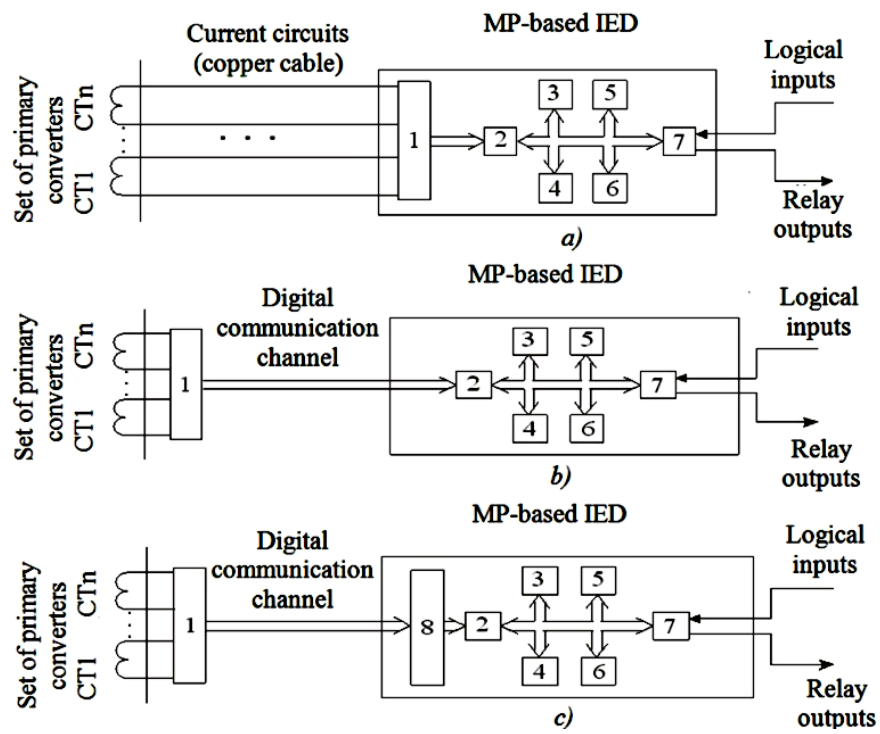

1 - analog/digital converter (ADC); 2 - central microprocessor; 3 - RAM-type memory unit; 4 - Hard Disktype ROM; 5 - flesh-memory-type programmable memory devices; 6 - Wi-fi and Ethernet technology-based signal transmission device; 7 - logical inputs/outputs; 8 - digital/analog converter (DAC)

Figure 1. Structural diagrams of signal conversion in MP-based protections 
Since MP-based protection units are multifunctional (act as several protection devices simultaneously), ADC can have several dozens of connected primary converters (from one to a high number of CTs) that take measurements for each protection kind with further analog signal sampling in ADC and its transmission for processing to the central MP-based device.

The most widely spread design version of MP-based protection systems is the variant where ADC unit is an integral structural component of the relay protection IED and makes a single whole with it (having a single enclosure), Figure 1(a). With such a version, impact of external electromagnetic radiation on current circuits increases, which can lead to distortion of the signal received from instrument CT by ADC unit and, consequently, to protection system malfunction.

Placing ADC in direct proximity to primary instrument converters (CT), Figure 1(b), makes it possible to prevent CT secondary side voltage increases in case of accidental disconnection of current circuits as a result of an emergency or improper actions of personnel. However, use of an extended digital channel (which is most often represented by a coaxial cable or shielded twisted pair) in conditions of distribution networks of large industrial enterprises does not seem possible as protection against mechanical damage of the digital communication channel cannot be enabled.

Double-conversion circuitry (Figure 1(c)) uses ADC and further digital signal conversion back to analog one for electromechanical protection devices by means of digital/analog converters (DAC). Such circuitries became wide spread at enterprises with a large number of kinds of protection devices in use. The main aim of using double-conversion circuitry is to enable recording of pre-emergency and emergency power system conditions (for further analysis), even if the protection that operated is of electromechanical type. The main drawback of this system is its high cost caused by simultaneous use of DAC and ADC units.

In design terms, MP-based relay protection units represent a set of planar printed circuit boards that are assembled like a PC. There is a motherboard, with connectors for the whole set of standard boards, on the enclosure bottom or sidewall. Such a circuit board also contains a system bus used as a interconnecting link for information transmission between MP-based unit components.

An MP-based protection unit, by all means, contains the following units (Figure 1):

a. A microprocessor-containing board;

b. A board enabling recording of processes that take place in memory of volatile and non-volatile types;

c. A system of transmitting signals using wireless communications;

d. A system of logical inputs/outputs used for programming mp-based protection operation modes;

e. Outputs of control signal for relays under control.

According to many specialists [1], [11]-[22], increased requirements are imposed on quality of the signal received by MP-based protection relays. The signal should not contain noise, it should stay only within its certain frequency range. Digital protections that transmit information to ADC unit not directly from primary converters but from filters or digital filters have a great advantage. Use of the latter enables reducing negative impact of powerful electromagnetic fields and ensuring stable operation of protection systems.

In some publications [1], [3]-[14] suggestion is made that for microprocessor relays it is desirable "to use more perfect current transducers than current transformers". An example of such current transducer is the Rogowski coil [1]-[21]. In this case there is no need for an integrating filter. It can be realized in the microprocessor software as a dedicated program.

\section{DIGITAL FILTER BASICS}

The theory of digital filters was developed soon after appearance of the first computers [22] and was included into course books [23], [24]. Digital filters can be divided into recursive and non-recursive ones [25], [26]. A non-recursive filter sums up a certain number of input values. Therefore its transfer function will have a numerator only. In a real device the delay line has a finite number of elements, so the impulse response will also be finite. Therefore non-recursive filters also have another name - filters with finite impulse response (FIR filters). The number of input values used to obtain the output signal is called the order of a filter.

A recursive filter sums up a certain number of input values and, in the general case, another number of output values. In this case the transfer function will have both numerator and denominator. Division of the numerator polynomial by denominator polynomial yields an infinite series of terms, so the pulse response of the filter is infinite. Therefore recursive filters also have another name - filters with infinite impulse response (IIR filters) [23]. In this article only IIR-filters will be considered and referred to as "digital filters". For a given frequency response such filters are simpler than FIR-filters as the latter require a much larger number of delay and multiplication elements [27]. For example, in [28] is shown that to obtain the desirable frequency response sometimes one needs FIR-filters of a very high order (several hundred or thousand). 
Two groups of digital filter synthesis are known: direct methods and methods based on use of analog prototype. In [29] is shown that among analog integrating filters the best properties has the second order filter having two equal real poles. Also are given recommendations how to provide the required accuracy and sufficient decay rate for transients in the filter itself. Therefore in the case under consideration it is quite reasonable to choose the second group methods.

Frequency response curves of digital filters are periodic (in contrast to those of analog filters). Their period is equal to the angular frequency of quantization, $\omega_{\partial}=2 \pi / T$, where $\mathrm{T}$ is the period (interval) of quantization. Therefore it is impossible to create a digital filter that is absolute identical to the analog prototype. There are different methods of synthesizing a digital filter to some extent corresponding to its analog counterpart.

\section{ANALYSIS OF DIGITAL FILTERS FOR MICROPROCESSOR RELAYS}

\subsection{Methods of synthesizing a digital filter}

During elaboration of material for this article 12 methods of synthesizing a digital filter basing on its analog prototype (second order integrating filter) were compared. The above methods include the following:

a. Method with standard z-transform as described in [24], [30];

b. Method with matched $\mathrm{z}$-transform as described in [30];

c. A group of methods with bilinear z-transform [31], [32].

It was found that at equal quantization period $\mathrm{T}$ the measurement errors of the filters synthesized using the first two methods are larger than those of filters synthesized using the last group of methods. This shortcoming is explained by overlapping of identical frequency responses shifted by $\omega_{\partial}$. The last group of methods does not have such a drawback.

If a bilinear transform method is used each term of the numerator and the denominator of initial transfer function (corresponding to the analog prototype) is divided by $s^{n}$, where $\mathrm{n}$ is the filter order. In this case negative powers of $\mathrm{s}$ will occur both in the numerator and the denominator. Then the following substitution is made:

$$
s^{-1}=\frac{T}{2} \frac{1+z^{-1}}{1-z^{-1}}, \quad z^{-1}=\exp \left(-s_{z} T\right)
$$

or

$$
s=\frac{2}{T} t h\left(\frac{s_{z} T}{2}\right)
$$

where $s^{-1}$ is Laplace transform of integration, $z^{-1}$ is Laplace transform of delay by quantization period $\mathrm{T}$, and $\mathrm{z}$ - the variable of Laurent transform [30] (now this transform is called "z-transform"). It can be seen that equation (1) describes the operation of numerical integration by method of trapezoids. Substituting $s=j \omega$ and $s_{z}=j \omega_{z}$ in (2) will yield:

$$
\omega=\frac{2}{T} \tan \left(\frac{\omega_{z} T}{2}\right) \text { or } \quad \omega_{z}=\frac{2}{T} \arctan \left(\frac{\omega T}{2}\right)
$$

where $\omega$ is the angular frequency of the analog prototype, and $\omega_{z}$ is the angular frequency of the digital filter. It can be seen that the frequency-response characteristic becomes more and more flattened with growth of angular frequency $\omega_{z}$. At Nyquist frequency [23], [30] that is equal to $\pi / T$ (two times smaller than the quantization angular frequency) this characteristic reaches the value of the analog prototype response at infinite frequency $\omega$. There is no aliasing in this case; however another shortcoming arises due to nonlinear deformation of frequency scale.

In the low frequency domain when $\omega$ is much smaller than Nyquist frequency the arctangent is approximately equal to its argument, so $\omega z \approx \omega$. In this case the frequency responses of digital and analog 
filters almost coincide. Then as the frequency rises the frequency response of the digital filter becomes more and more "compressed" as compared with its analog prototype.

In early digital equipment the quantization frequency was low. As a result the accuracy of calculation of multiple integrals having Laplace transforms described as $s^{-n}$ was also low. Therefore there appeared some methods in which terms $s^{-n}$ were not calculated as n-fold multiplication of the right term of Equation (1). Instead, other (more complex) expressions employing numerical methods of multiple integration were used.

\subsection{Use of boxer-thaler method and z-transform}

Among such methods the most known one is Boxer-Thaler method [33]. In [28] is given a table of multiple integrator transfer functions according to this method for orders up to five inclusive. In [34],[35] are provided results of study of second-order digital integrators derived from transfer function $W_{\text {in } 2}(s)=1 / s^{2}$ using bilinear transform $H_{\text {in } 2}(z)$, Boxer-Thaler transform $H_{i n 2 B T}(z)$, and Ragazzini-Bergen transform [36] $H_{\text {in } 2 R B}(z)$ :

$$
H_{i n 2}(z)=\frac{T^{2}}{4} \frac{\left(1+z^{-1}\right)^{2}}{\left(1-z^{-1}\right)^{2}}, H_{i n 2 B T}(z)=\frac{T^{2}}{12} \frac{1+10 z^{-1}+z^{-2}}{\left(1-z^{-1}\right)^{2}}, H_{i n 2 R B}(z)=\frac{T^{2}}{6} \frac{1+4 z^{-1}+z^{-2}}{\left(1-z^{-1}\right)^{2}}
$$

Judging by the frequency responses Ragazzini-Bergen transform is inferior to Boxer-Thaler transform in terms of the frequency range with acceptable accuracy. At an allowable error of $1 \%$ the upper boundary of this range for second-order integrator derived using Ragazzini-Bergen transform is only $\sqrt{2}$ times higher than for a similar integrator derived using the bilinear transform. For the second-order integrator derived from Boxer-Thaler transform this factor is equal to 5 i.e. it is 3.5 times higher than for RagazziniBergen transform. Therefore the latter transform is not considered below.

To obtain the system function of the digital integrating filter let us use the second-order transfer function [22]. Selecting the plus sign before the right member of this formula we will obtain the transfer function of the filter in normalized form:

$$
\bar{W}_{2}(\bar{s})=\frac{\bar{s}+2 \bar{a}}{(\bar{s}+\bar{a})^{2}}
$$

where $\bar{a}=a / \omega_{1}$ is relative value of $W_{n}(s)$ pole modulus [30]. The lower is a, the lower is the measuring error, but its minimum value-based constraint is imposed on selection of this pole. The matter is that the filter transfer function poles are inverse values with respect to its $T$ time constants. It means that $a=1 / T^{.}$[30].

Unlike in case of Boxer-Thaler method if the bilinear z-transform is used the operation of reduction to expression with negative powers of $\bar{s}$ can be eliminated. Then basing on [24], [30], we obtain:

$$
\bar{s}=\frac{2}{\bar{T}} \frac{1-z^{-1}}{1+z^{-1}},
$$

where $\bar{T}$ is the relative value of the quantization period

$$
\bar{T}=T \omega_{1}
$$

where $\omega_{1}$ is the angular frequency of the first harmonic of measured current $\left(\omega_{1}=100 \pi \mathrm{rad} / \mathrm{s}\right.$ for the case considered). After substitution of (7) into (6) and further simplification the normalized system function of the second-order filter is obtained: 


$$
\bar{H}_{2}(z)=\frac{m_{0}+m_{1} z^{-1}+m_{2} z^{-2}}{n_{0}+n_{1} z^{-1}+n_{2} z^{-2}} .
$$

The factors of the nominator and denominator of the system function are determined by the following expressions:

$$
\begin{aligned}
& m_{0}=2 \bar{T}+2 \bar{T}^{2} \bar{a} ; \quad m_{1}=4 \bar{T}^{2} \bar{a} ; \quad m_{2}=-2 \bar{T}+2 \bar{T}^{2} \bar{a} . \\
& n_{0}=4+4 \bar{T} \bar{a}+\bar{T}^{2} \bar{a}^{2} ; \quad n_{1}=-8+2 \bar{T}^{2} \bar{a}^{2} ; \quad n_{2}=4-4 \bar{T} \bar{a}+\bar{T}^{2} \bar{a}^{2} .
\end{aligned}
$$

If Boxer-Thaler method is used the nominator and the denominator are divided by $\bar{s}^{2}$, that yields:

$$
\begin{aligned}
& \bar{W}_{2}(\bar{s})=\frac{\bar{s}^{-1}+2 \bar{a} \bar{s}^{-2}}{1+2 \bar{a} \bar{s}^{-1}+\bar{a}^{2} \bar{s}^{-2}} . \\
& \text { Substitution of } \bar{s}^{-1} \text { with } \frac{\bar{T}}{2} \frac{1+z^{-1}}{1-z^{-1}} \text { and } \bar{s}^{-2} \text { with } \frac{\bar{T}^{2}}{12} \frac{1+10 z^{-1}+z^{-2}}{\left(1-z^{-1}\right)^{2}} \text { in (9) yields the system }
\end{aligned}
$$

function of the second-order digital filter, $\bar{H}_{2 B T}(z)$. After simplification this function will be of the form:

$$
\bar{H}_{2 B T}(z)=\frac{m_{0 B T}+m_{1 B T} z^{-1}+m_{2 B T} z^{-2}}{n_{0 B T}+n_{1 B T} z^{-1}+n_{2 B T} z^{-2}} .
$$

The factors of the nominator and denominator of function $\bar{H}_{2 B T}(z)$ are determined by expressions:

$$
\begin{aligned}
& m_{O B T}=6 \bar{T}+2 \bar{T}^{2} \bar{a} ; m_{1 B T}=20 \bar{T}^{2} \bar{a} ; m_{2 B T}=-6 \bar{T}+2 \bar{T}^{2} \bar{a} . \\
& n_{0 B T}=12+12 \bar{T} \bar{a}+\bar{T}^{2} \bar{a}^{2} ; n_{1 B T}=-24+10 \bar{T}^{2} \bar{a}^{2} ; n_{2}=12-12 \bar{T} \bar{a}+\bar{T}^{2} \bar{a}^{2} .
\end{aligned}
$$

Basing on functions (9) and (13) can be found the difference equations determining conversion of the input signal into the output one. The frequency characteristics corresponding to transfer function (6) of the analog filter and system functions (9) and (13) of the digital filters have differences as well as the errors of measurements with these filters.

\subsection{Signal sampling and quantization in digital filters}

Digital filters have specific features related to sampling of the input signal and its amplitude quantization [22]-[28], [37]-[39]. The first one is illustrated by Figure 2.

An analog-to-digital converter (ADC) contains a sample-and-hold circuit that operates as a switch driven by short pulses occurring with a period $\mathrm{T}$. When the switch is closed a pulse with amplitude equal to the instantaneous voltage on the ADC input (thin line in Figure 2(a)) appears on the output of the sampleand-hold circuit. The duration of such pulses is equal to the sampling period of the input analog signal.

The ADC also contains a quantizer converting the sample into a number with finite resolution. Then the sequence of numbers comes to the microprocessor performing the function of digital filter [30]. This sequence of numbers is shown in Figure 2(b) as the thick line curve. By the instant the next pulse driving the sampling-and-hold circuit appears the analog signal changes. This change causes ADC error to appear. One can see that there is both absolute value error and phase error as the first harmonic of the ADC output signal 
lags in phase behind the input signal. The graph of this error is shown in Figure 2(b). It constitutes a sequence of pulses limited by sine wave envelope. Since in Figure 1a the input signal has unity amplitude this graph represents the ADC relative error.

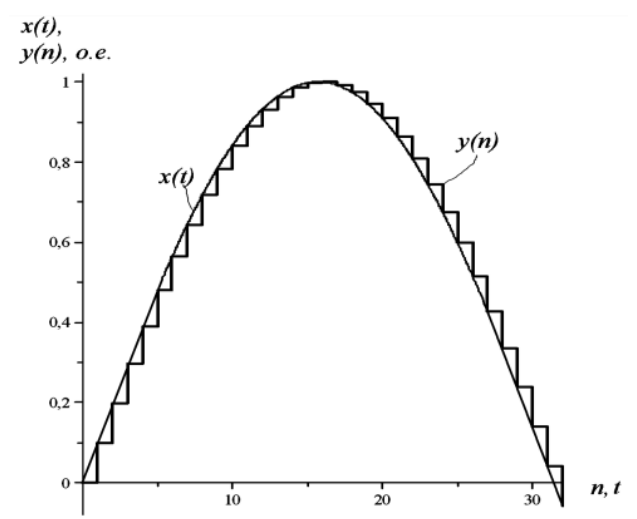

(a)

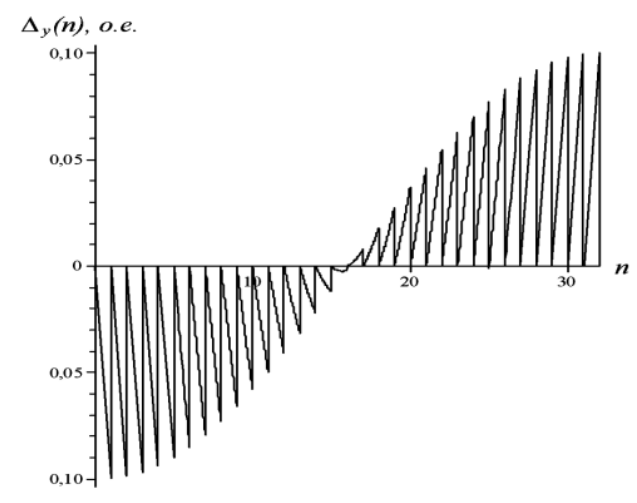

(b)

(a) Thin line - initial signal, thick line - ADC output signal; error in ADC "output" signal;

(b) $\mathrm{n}$ is the pulse number

Figure 2. Sampling of sine wave signal with unity amplitude

According to Figure 2 the more is the derivative of the input signal at the instant of the switch operation (in the sample-and-hold circuit) the more are the peak values of the error due to quantization of this signal. Consequently the peak of error corresponds to zero-crossing of the input signal. If period $\mathrm{T}$ is short the first remarkable limit is valid [40]-[43], so this maximum error is equal to normalized sampling period $\bar{T}=\omega_{1} T$.

The sampling period should be chosen so that the Nyquist frequency is at least three times higher than the angular frequency of the highest-order harmonic in the current to be measured. Hence the following calculation formula can be derived:

$$
T<\frac{1}{3 \omega_{1} n_{\max }} \rightarrow T \omega_{1}<\frac{1}{3 n_{\max }} \rightarrow \bar{T}<\frac{1}{3 n_{\max }},
$$

where $n_{\max }$ is the highest harmonic order.

As indicated in [24], [30], [44], $n_{\max }=40$. Therefore the condition $\bar{T}<0,0083$ must be met. Selection of $\bar{T}$ can be made more precise by studying the influence of this parameter on the additional error relative to the analog prototype.

\subsection{Comparison of frequency responses of analog and digital filters}

Let us compare the frequency responses of the analog prototype and the second-order digital filter. To obtain the frequency characteristic of the analog filter we should make substitution $\bar{s}=j \bar{\omega}$ in (6). To obtain the frequency characteristic of the digital filter - substitute $\bar{a}$ and $\bar{T}$ for their numerical values in (10) and (11) for the bilinear filter or in (14) and (15) for Boxer-Thaler filter. Then we make substitution $z^{-1}=\exp (-j \tilde{\omega} \bar{T})$ in (9) for the first filter type or in (13) for the second type.

The calculations were made for two values of parameter $\bar{a}: 0,0055$ - for the largest step-response error of $5 \%$ and 0,0027 - for the largest step-response error of 2,5\%. In Figure 3(a) are shown the dependencies of bilinear filter frequency response relative error vs. the relative frequency at $\bar{a}=0,0027$. 
(They almost coincide with the similar dependencies for $\bar{a}=0,0055)$. The analog filter frequency response is used as reference.

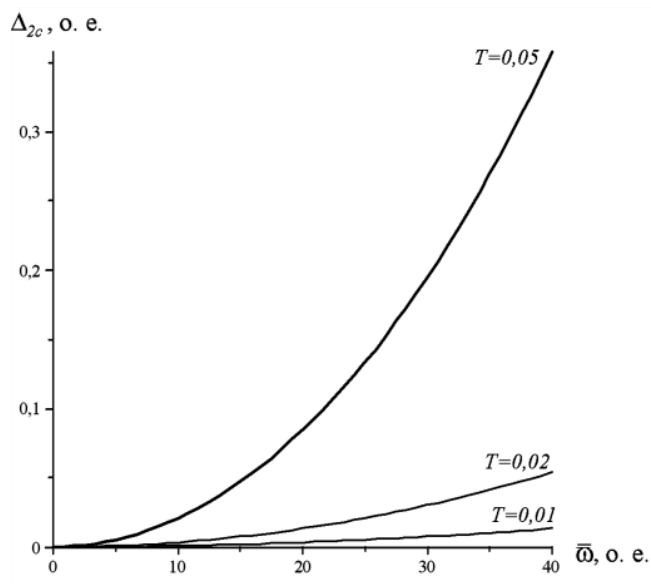

(a)

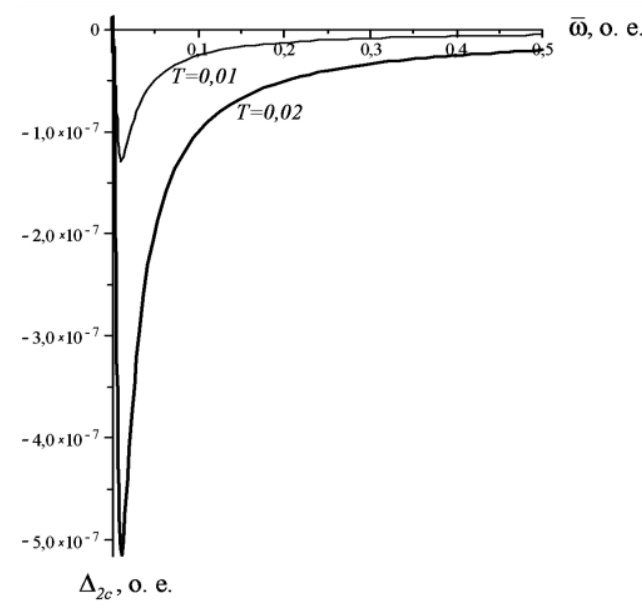

(b)

Figure 3. (a) Relative errors of bilinear filter frequency response for given $\bar{T}$ at $\bar{a}=0,0027$; (b) relative difference of frequency responses for bilinear filter and Boxer-Thaler filter for given $\bar{T}$ at $\bar{a}=0,0027$

This figure confirms the validity of condition $\bar{T}<0,026$ : at $\bar{\omega}=40$ the filter error at $\bar{T}=0,05$ is too large $(36 \%$, ) at $\bar{T}=0,02$ it drops to $5,4 \%$, and at $\bar{T}=0,01$ it is only $1,3 \%$. This is due to the fact that the Nyquist frequency rises with reduction of $\bar{T}$ farther and farther from $\bar{\omega}=40$.

As can be seen from Figure 3(b) the frequency responses of the bilinear filter and Boxer-Thaler filter almost coincide - the difference is negligibly small and is noticeable only at very low frequencies. At $\bar{a}=0,0055$ the ordinates of the graphs rise approximately five times but even in this case the difference is small. For correct calculation of the characteristics shown in Figure 3(b) we need at least 18 significant decimal places at $\bar{T}=0,02$ and at least 19 significant decimal places at $\bar{T}=0,01$.

In Figure 4(a) illustrates the fundamental difference between the frequency response of analog filters (thick line) and digital filters (thin line). The graph of frequency response of bilinear filter corresponds to $\bar{T}=0,02$ and $\bar{a}=0,0027$. It is very close to the graph corresponding to the different combination of parameters, $\bar{T}=0,02$ and $\bar{a}=0,0055$.

The frequency response of analog filters similar to the filters considered goes to zero at infinite frequency. In this case the main branch of the frequency characteristic corresponds to values of $\bar{\omega}$ ranging from zero to the Nyquist frequency. This frequency range is called "Nyquist interval" [30]. The closer is the frequency to the Nyquist frequency the lower is the frequency response of the digital filter relative to the analog prototype. At Nyquist frequency is reaches zero as shown in Figure 4(b) (thick and thin lower lines).

At further increase of frequency the frequency response of the digital filter rises. The frequency response curve in the range $\bar{\omega}_{N} \leq \bar{\omega} \leq \bar{\omega}_{\partial}$ where the angular sampling frequency $\bar{\omega}_{\partial}=2 \bar{\omega}_{N}$ is a "mirror reflection" of the main branch corresponding to the interval $0 \leq \bar{\omega} \leq \bar{\omega}_{N}$ relative to line $\bar{\omega}=\bar{\omega}_{N}$. As the frequency response at the Nyquist frequency is zero there is no aliasing between the main branch of the characteristics and its "mirror reflection". The main interval of the frequency characteristic corresponding to the interval $\bar{\omega}_{N} \leq \bar{\omega} \leq \bar{\omega}_{\partial}$ has an infinite number of copies shifted (relative to this interval) by $\pm n \bar{\omega}_{\partial}, \quad n=1 \ldots \infty$. 


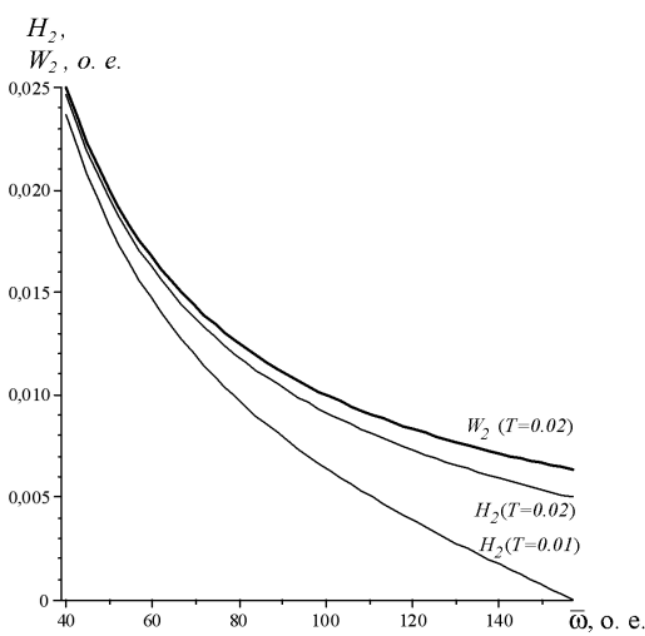

(a)

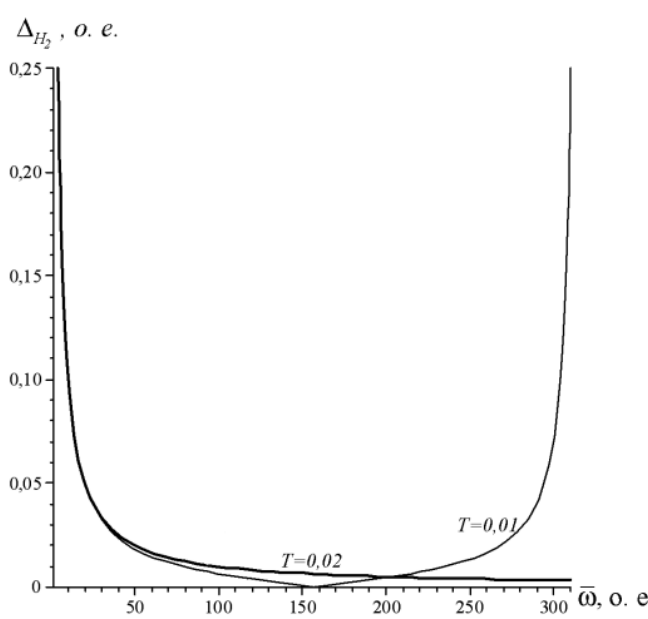

(b)

Figure 4. (a) Frequency response of analog filter and digital bilinear filters at $\bar{a}=0,0027$ for given $\bar{T}$; (b) relative difference of frequency responses for bilinear filter and Boxer-Thaler filter at $\bar{a}=0,0027$ for given $\bar{T}$

In Figure 5 are shown the initial part of the main branch of the filter frequency response curve and the graph of $1 / \bar{\omega}$ representing the frequency response of ideal integrator (thick line). Comparison of Figure 5(a) and Figure 5(b) allows to conclude that the larger is the value of $\bar{a}$ the earlier the difference of digital filter frequency response becomes noticeable. The derivative of the measured current is converted into the output signal of the digital bilinear integrating filter by software according to the following expression corresponding to formula (9):

$$
\begin{aligned}
y_{\text {out }}[k]= & \frac{1}{n_{0}}\left(m_{2} y_{\text {in }}[k-2]+m_{1} y_{\text {in }}[k-1]+m_{0} y_{\text {in }}[k]\right)- \\
& -\frac{1}{n_{0}}\left(n_{2} y_{\text {out }}[k-2]+n_{1} y_{\text {out }}[k-1]\right),
\end{aligned}
$$

where $y_{\text {in }}[j]$ is the $\mathrm{j}$-th sample of the analog input signal that is, in the case under consideration, the normalized derivative of measured current $d i / d \theta$, and $y_{\text {out }}[j]$ is $\mathrm{j}$-th term of the filter output signal sequence. The factors in expression (17) are calculated using formulas (10) and (11). The first (corresponding to beginning of measurement) two members of the sequence are found using the following formulas:

$$
y_{\text {out }}[0]=\frac{m_{0}}{n_{0}} y_{\text {in }}[0], \quad y_{\text {out }}[1]=\frac{m_{1} y_{\text {in }}[0]+m_{0} y_{\text {in }}[1]-n_{1} y_{\text {out }}[0]}{n_{0}}
$$




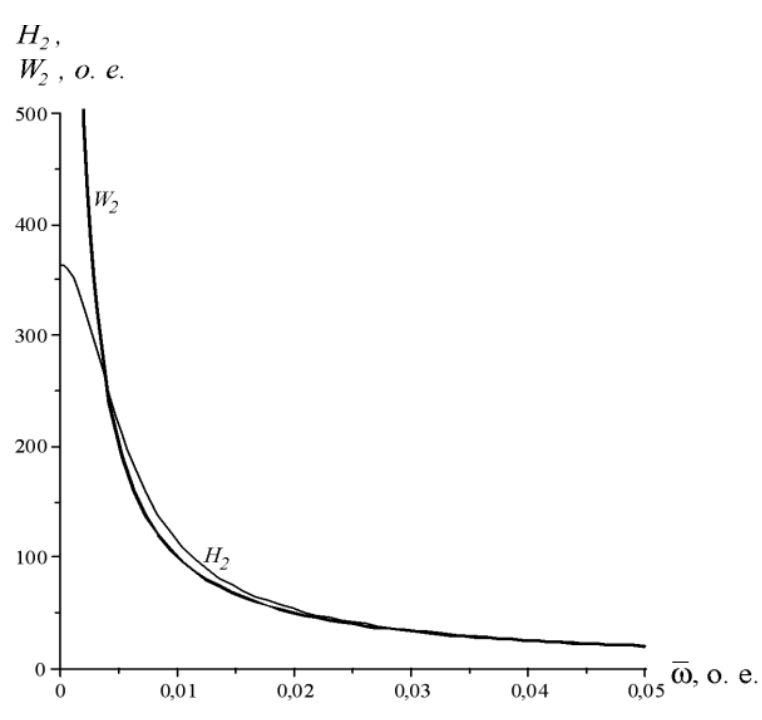

(a)

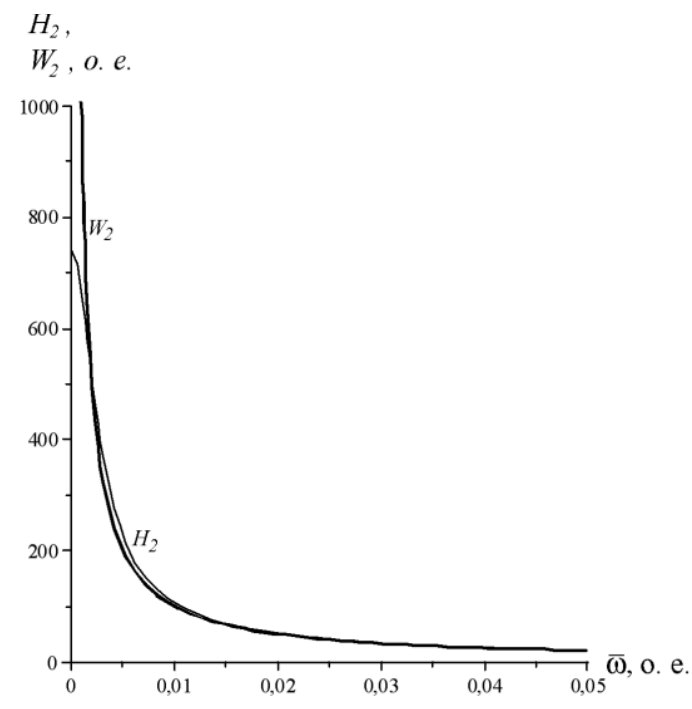

(b)

Figure 5. (a) Frequency response of ideal analog integrator (thick line) and of bilinear digital filters (thin line) for $\bar{T}=0,02$ : at $\bar{a}=0,0055$ and (b) at $\bar{a}=0,0027$

As shown in [22], the largest error of the analog filter occurs during measurement of transient current at $\alpha=25$ and $\beta= \pm \pi / 2$. Therefore for determination of digital filter calculation error let us use the following formula for normalized reference current:

$$
i=\cos \theta-\exp (-\theta / 25)
$$
the formula:

Then the derivative of this current, i.e. the normalized input of the integrating filter, is described by

$$
\frac{d i}{d \theta}=y_{i n}=-\sin \theta+\frac{1}{25} \exp (-\theta / 25)
$$

Making the substitution $\theta=j \bar{T}, j=0 \ldots N$, where $\mathrm{N}$ is the quantity of input sequence members, we will obtain array $y_{i n}[j]$ of input discrete values. Using this array and the two initial values calculated with (18) according to routine (17) the array of output discrete values, $y_{\text {out }}[j]$, is calculated.

In the same way as with array $y_{i n}[j]$, the array of measured current $i[j]$ is created basing on expression (19). Differences $\Delta_{i}=i[j]-y_{\text {out }}[j]$ form the array of errors of current measurement with the second-order bilinear filter. The curve of this error calculated for $\bar{a}=0,0055$ at $\bar{T}=0,01$ is shown in Figure 6(a) while that at $\bar{T}=0,02$ in Figure 6(b).

These curves are calculated using 11 significant decimal places. Dependencies of maximum full error and number of periods elapsed from the beginning of the transient vs. number of significant decimal places at $\bar{T}=0,02$ are shown in Table 1 . Also are shown the corresponding values for the second-order analog filter. 


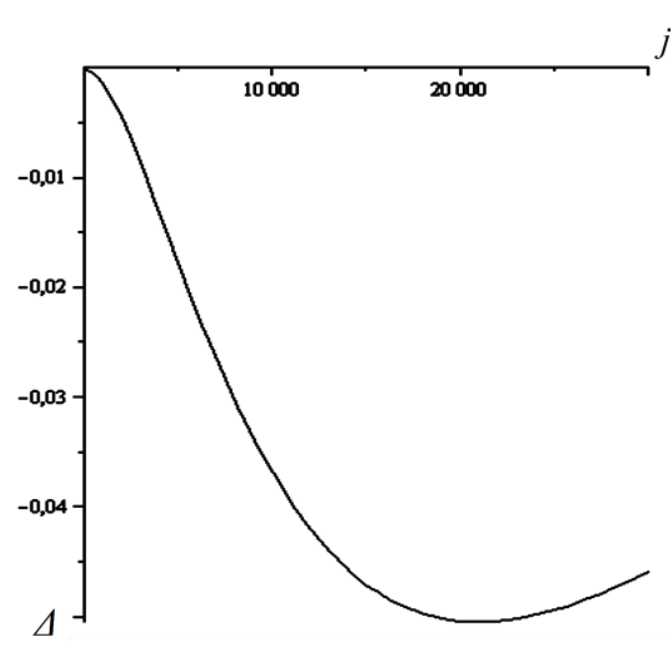

(a)

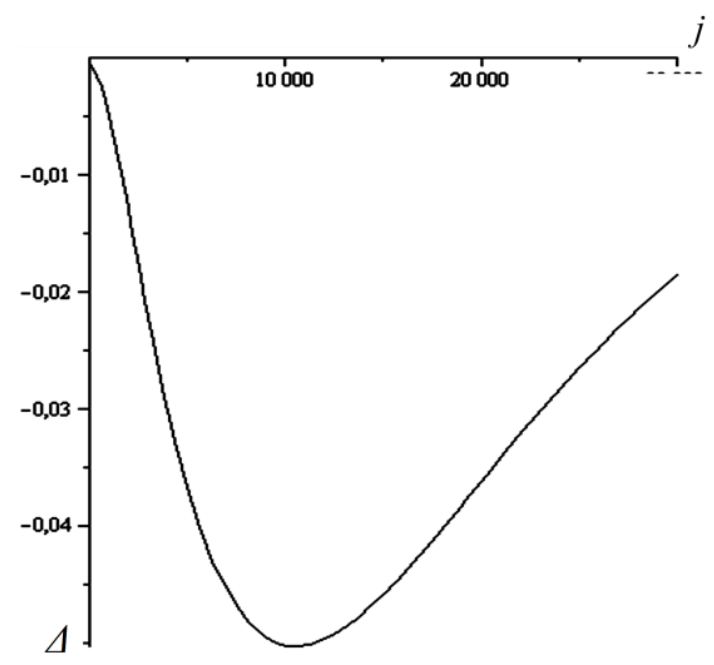

(b)

Figure 6. (a) Errors of transient current measurement vs. sample number for second-order bilinear filters for $\bar{a}=0.0055$ : at $\bar{T}=0.01$ and (b) at $\bar{T}=0,02$

Table 1. Parameters of Second-order Analog and Bilinear Digital Filters during Measurement of Current Corresponding to Equation (19)

\begin{tabular}{ccccccccc}
\hline & \multicolumn{8}{c}{ Digital filter, } \\
Parameters of maximum error & Analog & \multicolumn{6}{c}{ number of significant decimal places } \\
& filter & 7 & 8 & 9 & 10 & 11 & 12 \\
\hline $\begin{array}{c}\text { Number of periods till maximum of full } \\
\text { error, } N \text { max }\end{array}$ & 33,7 & 83,0 & 30,1 & 31,2 & 33,5 & 33,5 & 33,5 \\
Maximum full error, \% & 5,0 & 11,7 & 8,54 & 5,79 & 5,06 & 5,03 & 5,03 \\
\hline
\end{tabular}

It can be seen that as the number of significant places grows the error steadily declines and reaches its final value at 11 significant places. According to the data in Table 1 it is enough to have 10 significant places. In this case the curve of measurement error is smooth and "quantization noise" [22]-[28], [45]-[48] in the signals does not appear. If the said condition is met both analog and digital filters have virtually the same maximum error and the same time it is reached.

At 10 significant decimal places and $\bar{T}=0,01$ the maximum error has the same value as at $\bar{T}=0,02$. This assertion is confirmed by the curves shown in Figure 6 .

Dependencies of maximum full error and number of periods elapsed from the beginning of the transient vs. number of significant decimal places at $\bar{T}=0,02$ for second-order Boxer-Thaler filters are shown in Table 2.

Table 2. Parameters of Second-order Analog and Boxer-Thaler Digital Filters during Measurement of Current Corresponding to Equation (19)

\begin{tabular}{|c|c|c|c|c|c|c|c|}
\hline \multirow[t]{2}{*}{ Parameters of maximum error } & \multirow{2}{*}{$\begin{array}{l}\text { Analog } \\
\text { filter }\end{array}$} & \multicolumn{6}{|c|}{$\begin{array}{c}\text { Digital filter, } \\
\text { number of significant decimal places }\end{array}$} \\
\hline & & 7 & 8 & 9 & 10 & 11 & 12 \\
\hline \multicolumn{8}{|l|}{ Number of periods till maximum } \\
\hline of full error, $N_{\max }$ & 33,7 & 114,5 & 29,6 & 50,5 & 34,0 & 33,5 & 33,5 \\
\hline Maximum full error, $\%$ & 5,0 & $-14,8$ & 7,95 & 1,92 & 4,71 & 5,03 & 5,03 \\
\hline
\end{tabular}

It can be seen that the data shown in Table 1 and Table 2 coincide if the number of significant places is 11 or 12. This means that in such cases the Boxer-Thaler filter has no advantage over the bilinear filter. At 
10 significant places the maximum error of the Boxer-Thaler filter is 1,07 times smaller than with bilinear filter. However in this case there appears some subharmonic "quantization noise" with a swing of 0,001\% and a period of approximately 0,8 of the period of the measured current periodic component.

At 9 significant decimal places the maximum error with Boxer-Thaler filter is three times lower than with the bilinear filter. However the time of reaching the maximum in this case is 1,7 times longer. The swing of "quantization noise" is 5 times larger than with 10 significant places. At seven significant decimal places the maximum error of Boxer-Thaler filter changes its sign relative to the maximum errors at larger numbers of significant places. This error has a larger value $(14,8 \%)$ and occurs 1,4 times later than with the bilinear filter.

Reduction of measurement error for a current waveform given by (19) at low number of significant decimal places as compared with the analog filter is alarming. It is quite possible that in case of measurement of currents for other types of transients the measurement error will on the contrary become larger than with the analog filter. Therefore for the application considered the bilinear digital filter can be chosen as the optimal solution.

Performance of any digital filter can be reliably evaluated only in case of input impacts approximating, by their kind, actual secondary current waveforms of primary instrument current converters (mostly represented by current transformers or other converters [3], [14]-[18]) the protection using the evaluated filter is connected to. For these purposes, a power system and MP-based (digital) protection current measuring element structural model was developed and reproduced in MicroCAP dynamic modeling environment. Calculations led to the conclusion that if the primary current signal contains exponentially damping aperiodic component the primary current converter magnetic core (Rogowski coil) gets saturated even with permissible primary current ratio and rated secondary load. This results in Rogowski coil secondary current distortion and appearance of higher harmonics therein, as well as multiple harmonics. Comparing filter responses [14], [21] to the above-mentioned impacts once again confirmed that selection of a digital bilinear filter, which enables determining input signal amplitude with smallest errors, is correct.

\section{RESULTS AND DISCUSSION}

In conditions of large industrial enterprises of Primorsky Kray (Russian Federation), MP-based protections are used only as standby ones. As an experiment, such a system was introduced at "Novoshakhtinskoye" mining enterprise in 2013. The structural diagram of this system is shown in Figure 7.

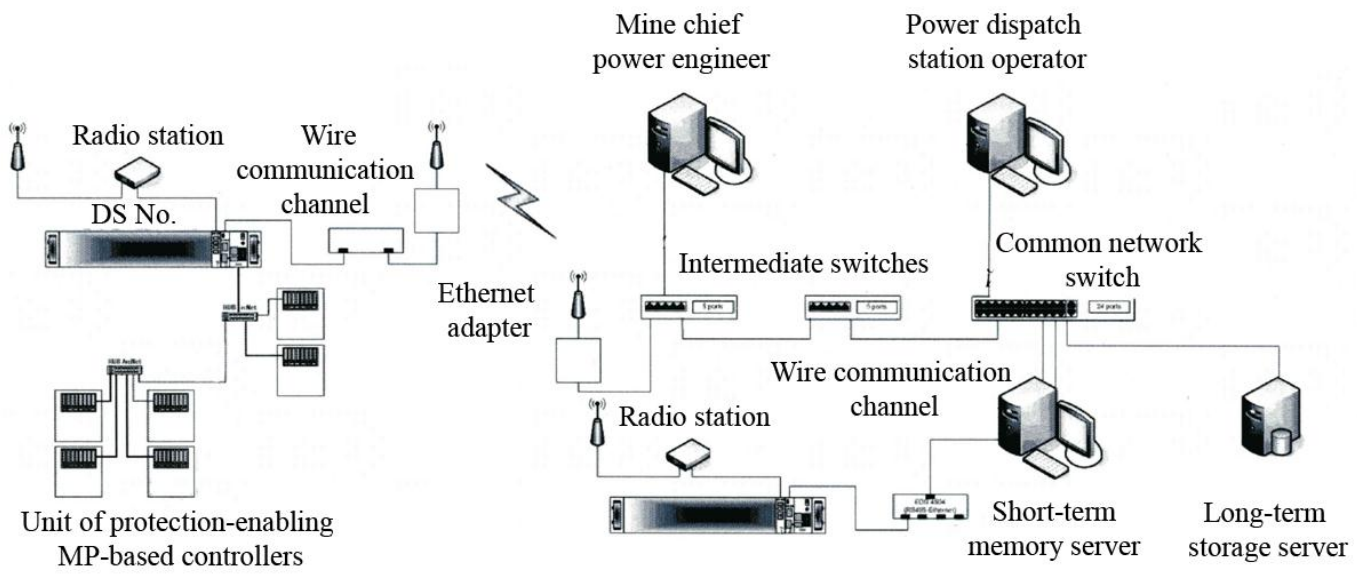

Figure 7. Structural diagram of a metering and monitoring system with MP-based protection functions

An automated monitoring and protection system has two levels. The first level includes MP-based controllers serving as standby protection. They are programmed for protection against emergency operation conditions and are installed at distribution stations (DS).

Information from controllers is transmitted using wireless communications to the central station that belongs to the second level. The central station includes an operational server that stores information about power network parameters and operation modes of all process power equipment in use at the enterprise for the duration of one day. After that information from the operational server is transmitted over a wire 
communication channel to the long-term storage server, which also receives information about the state of the main step-down substation (MSDS).

The second level also includes operator stations (displays) that display information for real-time monitoring of processes in the enterprise power network. Such stations are installed at the power engineering department operator room and at the chief power engineer of the enterprise. Based on the experience of using digital bilinear filters in MP-based IEDs (during four years), it can be pointed out that use of microprocessor protection as standby reduces the number of power equipment failures by average $50 \%$ per year. Table 3 contains the data that indicate the number of power equipment failures at "Novoshakhtinskoye" enterprise in the period from 2014 to 2017.

Table 3. Number of Power Equipment Failures at "Novoshakhtinskoye" Enterprise

\begin{tabular}{|c|c|c|c|c|}
\hline \multirow{2}{*}{ Protection kind } & \multicolumn{4}{|c|}{ Years } \\
\hline & 2014 & 2015 & 2016 & 2017 \\
\hline $\begin{array}{l}\text { Distribution stations using electromechanical protection } \\
\text { and MP-based protection as standby ( } 47 \text { electric loads) }\end{array}$ & 5 & 3 & 2 & 3 \\
\hline $\begin{array}{l}\text { Distribution stations only with electromechanical } \\
\text { protection (48 electric loads) }\end{array}$ & 7 & 8 & 5 & 6 \\
\hline Distribution stations without protection (39 electric loads) & 17 & 25 & 20 & 18 \\
\hline
\end{tabular}

It should be pointed out that the new integrated systems are MP-based, which entails some complexities in coordination of operation of digital devices and conventional electromechanical ones. Failure of protection relays to operate, primarily, is caused by unwanted tripping of protection systems by personnel. As a rule, there arise difficulties connected with established practices and ideas about relay protection of the enterprise power engineering department personnel and the need in additional personnel training. However, the main reason that relay protection devices fail to operate is distortion of signals received from primary converters, which leads to malfunctions or even breakdowns of MP-based information processing units.

According to the results of operation analysis of MP-based protection IEDs, there are situations when MP-based protection devices fail to operate or respond to the emergency conditions much slower than electromechanical ones. Such operation peculiarities of fast-acting MP-based protections are explained by a large number of signals MP-based relays operate with, as well as presence of electromagnetic disturbances that affect operation of digital systems.

\section{CONCLUSION}

In conditions of industrial enterprises, protection systems that simultaneously use electromechanical and standby MP-based relay protection devices have become widespread. Use of MP-based protection relays as standby protection enables reducing the number of failures of electrical machinery caused by emergency operation conditions.

At present, MP-based protections have some drawbacks. Digital relay protection systems, especially complex ones, for example, remote systems, do not always behave adequately in case of complex emergencies. Quite often complex MP-based protections malfunction or fail in actual complicated operating conditions of distribution networks of large industrial enterprises. If the relay is preliminarily tested in laboratory conditions with standard signals at its inputs, it operates accurately and reliably. The problem is that it is impossible to simulate, on that test bench, all possible signal combinations and distortions that can take place in reality beforehand. It is impossible to foresee such cases in relay design in advance, either.

Digital filters should be used to increase efficiency of MP-based protection IEDs. The conducted research showed that, among the examined digital filters, the digital bilinear filter demonstrates the best performance in close-to-real conditions.

\section{REFERENCES}

[1] L. Kojovic, "Rogowski Coils Suit Relay Protection and Measurement," IEEE Computer Applications in Power, vol. 10, no. 3, pp. 47-52, 1997.

[2] M. Faifer and R. Ottoboni, "An Electronic Current Transformer Based on Rogowski Coil," Instrumentation and Measurement Technology Conference Proceedings, IMTC 2008. IEEE, pp. 1554-1559, 2008.

[3] L. A. Kojovic, et al., "Innovative Differential Protection of Power Transformers Using Low-Energy Current Sensors," Industry Applications, IEEE Transactions, vol. 49, no. 5, pp. 1971-1978, 2013.

[4] A. Sharafi, et al., "Ultra-high-speed protection of parallel transmission lines using current travelling waves," IET Generation, Transmission \& Distribution, vol. 5, no. 6, pp. 656-666, 2011. 
[5] A. K. X. S. Campos, et al., "A new phasor estimation method for digital protective relays," Electric Power Systems Research, vol. 142, pp. 227-236, 2017.

[6] K. M. Silva and B. F. Küsel, "On combining wavelet-based designed filters and an adaptive mimic filter for phasor estimation in digital relaying," Electric Power Systems Research, vol. 92, pp. 60-72, 2012.

[7] B. Gu, et al., "High speed directional relaying algorithm based on the fundamental frequency positive sequence superimposed components," IET Generation, Transmission \& Distribution, vol. 8, no. 7, pp. 1211-1220, 2014.

[8] B. Osorno, "Application of Microprocessor Based Protective Relays in Power Systems," Industry Applications Society Annual Meeting, Conference on, 2009.

[9] V. E. Lane, "Digital protection and monitoring system for medium voltage substations and electric installations," Electricity Distribution. Part 1: Contributions. CIRED. 14th International Conference and Exhibition on (IEE Conf. Publ. No. 438), Conference on, 1997.

[10] L. Ayers, et al., "Protecting distribution substation assets - Modern protection schemes with microprocessor-based relays," Protective Relay Engineers, 2013 66th Annual Conference for, Conference on, 2013.

[11] G. Liao and J. Zhu, "Distribution Line Microprocessor Relay Protection System," Intelligent Computation Technology and Automation (ICICTA), 2010 International Conference on, 2010.

[12] B. V. Djokic, et al., "To what extent can rogowski coil current amplitude linearity be verified?" Precision Electromagnetic Measurements (CPEM), 2010. Conference on, pp. 541-542, 2010.

[13] Emerging Technologies Working Group and Fiber Optic Sensors Working Group, "Optical current transducers for power systems: A review,” IEEE Trans. Power Delivery, vol. 9, no. 4, pp. 1778-1788, 1994.

[14] D. B. Solovev, et al., "Analysis of modeling of current differential protection," International Journal of Power Electronics and Drive Systems, vol. 6, no. 3, pp. 423-428, 2015.

[15] D. B. Solovev, et al., "Instrument current transducers with Rogowski coils in protective relaying applications," International Journal of Electrical Power and Energy Systems, vol. 73, pp. 107-113, 2015.

[16] V. Skendzic and B. Hughes, "Using Rogowski coils inside protective relays," Protective Relay Engineers, 2013 66th Annual Conference, pp. 1-10, 2013.

[17] S. H. Horowitz and A. G. Phadke, "Power System Relaying," Third Edition, John Wiley \& Sons, Ltd, Chichester, UK, 2008.

[18] J. D. Bull, et al., "A new hybrid current sensor for high-voltage applications," IEEE Trans. Power Delivery, vol. 20, no. 1, pp. 32-38, 2005.

[19] W. Zhang, et al., "An improved technique for online PD detection on covered conductor lines," IEEE Trans. Power Delivery, vol. 29, no. 2, pp. 972-973, 2014.

[20] D. B. Solovev, et al., "Analyzing upcoming trends in development of current transducers for automatic equipment and relay protection: A Review," International Review of Electrical Engineering (IREE), vol. 10, no. 3, pp. 381-389, 2015.

[21] D. B. Solovev, et al., "Implementation of noise-immune Rogowski coils for busbar differential protection modernization," Electric Power Systems Research, vol. 140, pp. 965-975, 2016.

[22] F. P. G. Márquez and N. Zaman, "Digital Filters and Signal Processing," in Tech, Chapters published January 16, 2013 under CC BY 3.0 license, 2013.

[23] S. Theodoridis and R. Chellappa, "Academic Press Library in Signal Processing: Signal Processing Theory and Machine Learning," Academic Press, 2013.

[24] B. A. Shenoi, "Introduction to Digital Signal Processing and Filter Design," John Wiley \& Sons, Inc., Hoboken, NJ, USA, 2005

[25] A. M. El Nady, et al., "Power-quality improvement using Wiener filters based on a modular compensating strategy," IEEE Trans. Power Delivery, vol. 23, no. 2, pp. 841-849, 2008.

[26] M. Badoni, et al., "Variable forgetting factor recursive least square control algorithm for DSTATCOM," IEEE Trans. Power Delivery, vol. 30, no. 5, pp. 2353-2361, 2015.

[27] W. Rebizant, et al., "Digital Signal Processing in Power System Protection and Control," Springer Science \& Business Media, 2011.

[28] S. W. Smith, "The Scientist \& Engineer's Guide to Digital Signal Processing," California Technical Pub, 1997.

[29] A. Handkiewicz, et al., "VHDL-AMS in switched-current analog filter pair design based on a gyrator-capacitor prototype circuit," International Journal of Numerical Modelling: Electronic Networks, Devices and Fields, vol. 27 , no. 2, pp. 268-281, 2014

[30] A. M. Haghighi and D. Mishev, "Difference and Differential Equations with Applications in Queueing Theory," John Wiley \& Sons, Inc., Hoboken, NJ, USA, 2013.

[31] F. Li, et al., "Improved teager energy operator and improved chirp-Z transform for parameter estimation of voltage flicker," IEEE Trans. Power Delivery, vol. 31, no. 1, pp. 245-253, 2016.

[32] T. Noda, et al., "z -Transform-based methods for electromagnetic transient simulations," IEEE Trans. Power Delivery, vol. 22, no. 3, pp. 1799-1805, 2007.

[33] R. Boxer and S. Thaler, "A simplified method for solving linear and nonlinear systems," Proceedings of the IRE, vol. 44, no. 1, pp. 89-101, 1956.

[34] J. R. Ragazzini and A. R. Bergen, "A mathematical technique for the analysis of linear systems," Proceedings of the IRE, vol. 42, no. 11, pp. 1645-1651, 1954.

[35] J. C. Alfonso-Gil, et al., "Enhanced grid fundamental positive-sequence digital synchronization structure," IEEE Trans. Power Delivery, vol. 28, no. 1, pp. 226-234, 2012.

[36] A. R. Bergen and J. R. Ragazzini, "Sampled-data processing for feedback control," Electrical Engineering, vol. 73, no. 11 , pp. $980,1954$. 
[37] J. K. Hwang, et al., "Reduction of noise in measurements of phasor angles by using two digital filters," IEEE Trans. Power Delivery, vol. 29, no. 1, pp. 52-60, 2014.

[38] S. A. Saleh, et al., "The development of a d-q axis WPT-based digital protection for power transformers," IEEE Trans. Power Delivery, vol. 27, no. 4, pp. 2255-2269, 2012.

[39] R. A. Zadeh, et al., "Online estimation of distorted power system signal parameters," IET Generation, Transmission \& Distribution, vol. 4, no. 6, pp. 746-755, 2010.

[40] J. D. Ghosh, "How To Learn Calculus Of One Variable," New Age International Limited, vol. 1, 2004.

[41] V. S. Shipachev, "Higher mathematics," Mir Publishers, 1988.

[42] "Differential and integral calculus," lecture notes (Tel-aviv university), 2009.

[43] A. N. Sarwade, et al., "Reach and operating time correction of digital distance relay," International Journal of Electrical and Computer Engineering, vol. 7, no. 1, pp. 58-67, 2017.

[44] B. U. Rindhe, et al., "Implementation of Optical OFDM Based System for Optical Networks," International Journal of Electrical and Computer Engineering, vol. 4, no. 5, pp. 767-781, 2014.

[45] A. Rezaee, et al., "Digital Encoder Designing for Mobile Robot Control," International Journal of Electrical and Computer Engineering, vol. 4, no. 5, pp. 648-657, 2014.

[46] E. Alibašić, et al., "Transient Phenomena during the Three-Phase 300MVA Transformer Energization on the Transmission Network," International Journal of Electrical and Computer Engineering, vol. 6, no. 6, pp. 2499-2505, 2016.

[47] Azriyenni, et al., "Application of ANFIS for Distance Relay Protection in Transmission Line," International Journal of Electrical and Computer Engineering, vol. 5, no. 6, pp. 1311-1318, 2015.

[48] Nasaruddin, et al., "Improved Model of the Selection with Soft and Hard Combining Decoding Strategies for Multi-User Multi-Relay Cooperative Networks," International Journal of Electrical and Computer Engineering, vol. 6, no. 4, pp. 1766-1778, 2016.

\section{BIOGRAPHY OF AUTHOR}

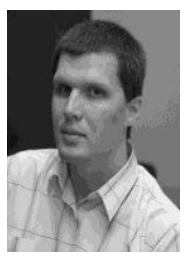

Denis B. Solovev is a graduate of Far Eastern State Technical University (Diploma with honours), Vladivostok, Russia. In 2011 he received a PhD in electric engineering. From 2011 to 2017 he took internship in the leading Russian and international universities and research laboratories: MATI Russian State Technological University named after K.E. Tsiolkovsky, Moscow; University of Maastricht, Maastricht (Netherlands); Laboratory of the Ministry of Energy of the United States of America, Seattle and Richland, Washington; Swiss Federal Institute of Technology Zurich, Zurich; Department of Electrical and Electronic Engineering Hong Kong University of Science and Technology, Hong Kong SAR, Hong Kong; Japan Society for the Promotion of Science Faculty of Engineering, Aichi Institute of Technology, Toyota, Japan. He is currently the head of the educational program "Innovatica" (cluster of energy and electronics) at the Engineering School of the Far Eastern Federal University, Vladivostok, Russia. Dr. Denis B. Solovev research interest covers the Electrical Energy and Power Technologies for smart energy conversion. He is a Senior Member of the IEEE, Member of IEEE Societies of IES, PES and serves as Reviewer for over 30 international journals. 